\title{
Comparison of Nepalese and Swedish Patients with Colorectal Cancer Regarding Tumor Suppressor Methylation in Tumor and Mucosa Samples
}

\author{
Bikal Ghimire1, Goran Kurlberg², Yogendra P Singh'1, Yvonne Wettergren² \\ 'Department of Gl and General Surgery, Maharajgunj Medical Campus, Tribhuvan University Teaching Hospital, \\ Institute of Medicine, Kathmandu, Nepal, ${ }^{2}$ Department of Surgery, Institute of Clinical Sciences, Sahlgrenska \\ Academy, University of Gothenburg, Gothenburg, Sweden
}

\section{Corresponding author:}

\section{Bikal Ghimire, MBBS, MS}

Department of Gl and General Surgery, Maharajgunj Medical Campus, Tribhuvan University Teaching Hospital, Institute of Medicine, Kathmandu, Nepal

Email: bikalghimire@gmail.com

\begin{abstract}
Introduction

Colorectal cancer (CRC) is common with wide variation in incidence. However, the incidence in younger adults is showing an increasing trend with rectal preponderance. Such cases appear to present in advanced stage with poorly differentiated histopathology and the trend is stronger in developing countries. The aim of the study was to compare the MLH1 and in matched mucosa and tumor samples obtained from the large gut of Nepalese and Swedish patients with CRC.
\end{abstract}

\section{Methods}

Patients diagnosed and operated for CRC at Tribhuvan University Teaching Hospital, Nepal $(n=39)$ matched for age with those operated at Sahlgrenska University Hospital, Sweden $(n=39)$ were included in the study. Demographics and clinicopathological data were compared. The tissue methylation status was compared in the Nepalese and Swedish populations and correlated to clinicopathological data.

\section{Results}

There were $56 \%$ men and $44 \%$ women in the study. Their mean age was 53 years with a range of 20 to 78 years. Colon cancer amounted to $70 \%$ and $60 \%$ in the Nepalese and Swedish patients respectively and the majority of lesions were on the right colon. The colorectal cancer was commonly of stage III (52\% and $38 \%$ ) in the Nepalese and Swedish group respectively. Most of the tumors were well differentiated (41\%) in the Nepalese population where as in Swedish patients it the differentiation was medium to low (52\% and $11 \%$ ). Methylation of MLH gene, in different CpG sites, when analyzed independently, showed that in about $18-20 \%$ of the cases, the degree of methylation is significantly higher in tumor tissue as compared to sites at least $10 \mathrm{~cm}$ away from the lesion.

\section{Conclusion}

Colon cancer is more common in men in Nepal with predominantly more on the right colon. Most of them present in advanced stage with higher MLH1 methylation.

Keywords: Colorectal cancer, MLH1, p14AR, methylation, MSI 